\title{
An Integrating of Clinical, Pathological, and Radiomic Approach to Predict the Microsatellite Instability in Rectal Carcinoma
}

\author{
Hang Yuan \\ Zhejiang Provincial People's Hospital \\ Peng Yu \\ Zhejiang Provincial People's Hospital \\ Jiankai Li \\ The People's Hospital of Anyang City \\ Niping Song \\ Zhejiang Chinese Medical University \\ Zi'ang Wan \\ Zhejiang Provincial People's Hospital \\ Bingchen Chen \\ Zhejiang Provincial People's Hospital \\ Shiliang Tu \\ Zhejiang Provincial People's Hospital \\ Yuguo Wei \\ GE Healthcare. \\ Yanqing Ma ( $\square$ 704180026@qq.com) \\ Zhejiang Provincial People's Hospital
}

\section{Research Article}

Keywords: Rectal carcinoma, Computed tomography, Microsatellite instability, Radiomics, nomogram

Posted Date: October 20th, 2021

DOI: https://doi.org/10.21203/rs.3.rs-957494/v1

License: @ (1) This work is licensed under a Creative Commons Attribution 4.0 International License. Read Full License 


\section{Abstract}

Objective: To develop an integrative model with clinical, pathological, and radiomic characteristics to predict the status of microsatellite instability (MSI) in rectal carcinoma (RC).

Methods: A cohort of 788 RCs with 97 high MSI status (MSI-H) and 691 microsatellite stable status (MSS) were enrolled. The clinical and pathological characteristics were recorded. The radiomic features were calculated after segmentation of volume of interests and then patients were divided into the training set and validation set with a random proportion of 7:3. The logistic models of simple clinical characteristics (LM-Clin), pathological characteristics (LM-Patho), and radiomic features (LM-Radio) were constructed to distinguish MSI-H from MSS. The relevant radiomic score was calculated. Finally, a integrative nomogram (LM-Nomo) including significant clinical, pathological characteristics, and radiomics was developed. The area under receiver operator curve (AUC) was calculated to evaluate the efficacy of prediction.

Results: The AUC of simple LM-Clin including variables of CEA and hypertension and LM-Patho including characteristics of gross type and lymph node metastasis ratio (LNR) was $0.584(95 \% \mathrm{Cl}, 0.549-0.619)$ and $0.585(95 \% \mathrm{Cl}, 0.550-0.619)$, which was lower than that of LM-Radio including 12 radiomic features with AUC of $0.737(95 \% \mathrm{Cl}, 0.675-0.799)$. The LM-Nomo contained CEA, hypertension, LNR, and radiomic score, and the AUC was $0.757(95 \% \mathrm{Cl}, 0.726-0.787)$.

Conclusion: The AUCs of LM-Clin and LM-Patho were disappointing and lower than that of LM-Radio. The LM-Nomo demonstrated the best performance in predicting MSI-H status.

\section{Background}

Colorectal carcinoma (CRC) is one of the most frequently diagnosed cancers and also the leading cause of cancer-related mortality ${ }^{[1]}$. Rectal carcinoma (RC) accounts for approximately 29\% of newly diagnosed CRC between 2012 to 2016 and is the most common type of CRC in people younger than 50 years old ${ }^{[2]}$. The prognosis of CRC depends on the biology and heterogeneity of the tumor ${ }^{[3]}$. Microsatellite instability (MSI) is an important biomarker of CRC with prominent diagnosis, prognosis, and prediction significance. Tumors detect loss of one or more mismatch repair (MMR) proteins testing by immunohistochemistry are considered to be high-MSI (MSI-H) ${ }^{[4]}$. Whereas those with intact MMR proteins are expected to be microsatellite stable or low-MSI (MSS or MSI-L). MSI is detected in about $15 \%$ of CRC patients and has emerged as a predictor of patient response to adjuvant chemotherapy ${ }^{[5]}$. MSI which shared clinicopathological characteristics distinctly different from the MSS ones has been reported to have a higher prevalence in stage II CRC ${ }^{[6]}$, and a better prognosis ${ }^{[7]}$.

Radiomics extracts the quantitative high-through image data from conventional images which was applied to improve diagnostic and predictive accuracy ${ }^{[8]}$, is gaining great attention in medical research. Previous studies indicated that computed tomography (CT) based ${ }^{[9]}$ or magnetic resonance (MR) based ${ }^{[10]}$ radiomics analysis helped to predict MSI status in CRC. To the best of our knowledge, there were only three articles that studied the MR-based ${ }^{[11,12]}$ and T2WI-based ${ }^{[13]}$ radiomic signature in predicting MSI phenotype of RCs. However, there was no CT relevant radiomic analysis in this field. It is meant to develop a non-invasive, reproducible CT radiomic approach to evaluate the MSI-H status of RCs. The purpose of this article was to construct and confirm an integrative model with clinical, pathological, and radiomics features to evaluate the status of MSI-H of RCs on the basis of three-phase CT images, preoperatively.

\section{Materials And Methods}

This retrospective study was conducted with the permission of the Medical Ethics Committee (No. 2020QT251) and in conformity to the Declaration of Helsinki. The informed consent was waived for this retrospective study.

\section{Patients selection}

There were 1103 patients who pathologically proved to be RCs from January 2015 to January 2021 after searching the surgical database in our hospital. The inclusion criteria were as follows: (a) patients were pathologically proved to be RCs, including classical adenocarcinoma, mucous adenocarcinoma, and signet-ring cell carcinoma. (b) all CT examinations were implemented within two weeks before surgeries. (c) in addition to the tumor occurring in the rectum, the tumors originated from the rectum to the adjacent colon sigmoideum were also recruited. The exclusion criteria were as follows: (a) patients received preoperative therapy including radiation, chemotherapy, or chemoradiotherapy. (b) patients with metachronous or recurrent cancer. (c) the lesion occurred in the ascending, descending, and sigmoid colon. In addition, cancers that originated from the junction of the rectosigmoid belonged to upper rectum carcinoma. (d) patients without MSI evaluation. 
Finally, a total of 788 patients with 97 MSI-H and 691 MSS were retrospectively enlisted in this analysis.

\section{Clinical and pathological characteristics of RC patients}

The baseline clinical variables for analysis included age, gender, body mass index (BMI), CT-displayed long diameter, tumor location (low RC refers to the lesion within $5 \mathrm{~cm}$ from anal margin, middle RC refers to the lesion is $5-10 \mathrm{~cm}$ from anal margin, high RC refers to the lesion is more than $10 \mathrm{~cm}$ away from the anal margin), carcinoembryonic antigen (CEA) with threshold values of $5.0 \mu \mathrm{g} / \mathrm{L}$, carbohydrate antigen 19-9 (CA19-9) with threshold values of $37.0 \mathrm{U} / \mathrm{mL}$, the history of smoking, drinking, diabetes, and hypertension. In addition, the tumor originated from the junction of the rectosigmoid region, and the distance to the anal margin greater than $10 \mathrm{~cm}$ was classified as high RC.

The pathological variables included gross type (protuberant, infiltrating, and ulcerative), histological type (classical adenocarcinoma, mucous adenocarcinoma, and signet-ring cell carcinoma), tumor differentiation degree (well, moderate, and poor), Ki-67-positive cells expression (67\%), T/N/M stage, vascular invasion, perineural invasion, lymph node count (LN), positive lymph node count (PLN), lymph node metastasis ratio (LNR, LNR=PLN/LN*100\%), and liver metastasis.

\section{Evaluation of MSI status}

The method of immunohistochemistry was used to test MMR proteins including MLH1, MSH2, MSH6, and PMS2. Tumors displaying a lack of one or more MMR proteins were collectively classified as defective mismatch repair (dMMR) and expected to be MSI-H, while those with intact MMR proteins were considered as proficient mismatch repair (pMMR) and estimated to be MSS or MSI-L. After referring to the revised Bethesda guideline for MSI, for clinical purposes, the MSI-L type for CRCs was be revised and categorized as MSS tumors ${ }^{[14]}$. Therefore, our study divided all the RC patients into two groups based on the MMR proteins: the MSI-H cohort and the MSS cohort.

\section{CT examination}

All the 789 RC patients were conducted three-phase examinations using 64/128 slices CT (Siemens, Somatom Definition AS). The threephase (unenhanced phase, arterial phase, and venous phase) CT examination was achieved by the method of computer-aid bolus tracking with a dose of $1.3 \mathrm{~mL} / \mathrm{kg}$ contrast media (iomeperol 350, GE Healthcare) at a rate of $3.0 \mathrm{~mL} / \mathrm{s}$ via a high-pressure injector. The arterial phase was scanned after $35 \mathrm{~s}$ of injection of iomeperol, and the venous phase was followed after $25 \mathrm{~s}$ of arterial phase. The specific parameters were as follows: $120 \mathrm{Kv}$ of tube voltage, $200 \mathrm{~mA}$ of tube current, $360 \mathrm{~mm}$ field of view, $64^{\star} 0.625 \mathrm{~mm}$ of collimation, $0.75 \mathrm{~s}$ of the rotation time, $5 \mathrm{~mm}$ of slice and interval thickness, and $300 \mathrm{HU}$ of window width, $40 \mathrm{HU}$ of window level.

\section{Tumor segmentation and radiomics features selection}

All the three-phase CT images were received from our picture archiving and communication system in DICOM format. After the standardization of original images using the software of "A.K. 3.0.0" (Artificial Intelligence Kit, GE Healthcare), the volume of interests (VOIs) were manually segmented in the software of "itk-SNAP 3.4.0" (http://www.itksnap.org/) by two radiologists with 7 and 10 years of experience, respectively (Figure 1a,b). The regions of necrosis, intraluminal air, non-invaded rectal wall, vessel, and peri-rectal fat were eliminated from contours of VOls.

Then the radiomic features of tumors were automatically calculated by A.K. software. The intraclass correlation coefficients (ICCs) of radiomic features from two radiologists were calculated, all the ICCs of radiomics features were greater than 0.75 , which was interpreted as of good agreement between different observers ${ }^{[15]}$. Therefore, the mean values of radiomic features from two radiologists were calculated for later research. Since the two sets of sample sizes were not balanced, the method of synthetic minority over-sampling technique (SMOTE) was used to balance them. SMOTE is a straightforward approach used for regulating the ratio between the unbalanced groups ${ }^{[16]}$. The cohort (97 MSI-H and $691 \mathrm{MSS}$ ) was randomly partitioned into a training set (68 MSI-H and $484 \mathrm{MSS}$ ) and a validation set (29 MSI-H and 207 MSS) with a proportion of 7:3. Hereafter, the methods of variance or Mann-Whitney U-test (ANOVA or MW), correlation analysis, and least absolute shrinkage and selection operator (LASSO) were performed to select optimal radiomic features. A 10-fold cross validation approach was used in both the training and validation cohorts to construct the model with the best performance. The detail of tumor segmentation and radiomics features selection were expounded in Supplement Material.

\section{Clinical, pathological, and radiomics models construction}

After radiomic features selection, this corresponding logistic model (LM-Radio) was constructed by the selected radiomic features and the radiomic score was acquired. The clinical and pathological characteristics were firstly analyzed by independent t-test or chi-square test. Then the clinical logistic model (LM-Clin) and pathological logistic model (LM-Patho) by corresponding significant variables were developed, respectively. The area under curves (AUCs) of the receiver operator curve (ROC) calculated by the Delong test were applied to assess the efficiency of all logistic models. Finally, an integrative clinical-pathological-radiomic nomogram (LM-Nomo) with radiomic score, significant clinical characteristics, and pathological parameters was constructed to evaluate the MSI-H status.

Page $3 / 13$ 


\section{Statistical analysis}

The methods for radiomics features selection including ANOVA or MW, correlation analysis, and LASSO were proceeded in R software (https://www.r-project.org/). The analysis of clinical and pathological characteristics was executed in SPSS software (https://spss64bits.en.softonic.com/) using the independent t-test or chi-square test. The ICCs were utilized to assess the consistency of VOI segmentation between two radiologists. The logistic models of LM-Clin, LM-Patho, and LM-Nomo with the method of entrance were developed in R software. The Delong test was carried out in MedCalc software (https://www.medcalc.org/), the corresponding AUC, 95\% confidence interval $(\mathrm{Cl})$ was recorded. A Hosmer-Lemeshow test was used to evaluate the goodness-of-fit and accuracy of the model. A twotailed $p$ value $<0.05$ indicated a statistical difference.

\section{Results}

\section{Baseline clinical and pathological characteristics}

The baseline clinical and pathological characteristics were outlined in Table 1. To the analysis of clinical characteristics, the variables of CEA $(p=0.043)$, history of smoking $(p=0.049)$, drinking $(p=0.026)$, and hypertension $(p=0.036)$ showed significant differences. The MSI-H cohort tended to have normal CEA (71.1\%) and was accompanied by the history of smoking (26.8), drinking (22.7\%), and hypertension (45.4\%) compared with MSS cohort. And there was statistical significance in the pathological characteristics of gross type ( $p=0.048)$, $T$ stage $(p=0.013)$, and LNR ( $p=0.041)$. The MSI-H cohort was more likely to occur in the T2/4 stage, to appear ulcerative type, and to have higher LNR than the cohort of MSS. 
Table 1

The baseline clinical and pathological characteristics.

MSI-H cohort( $\mathrm{n}=97)$

Clinical characteristics

Age (mean $\pm S D)$

Gender (female/male)

$\mathrm{BMI}\left(\mathrm{mean} \pm \mathrm{SD}, \mathrm{kg} / \mathrm{m}^{2}\right)$

Long diameter (mean $\pm S D, \mathrm{~mm})$

Location, $\mathrm{n}(\%)$

(low/middle/high)

CEA (normal/abnormal), $\mathrm{n}(\%)$

CA19-9 (normal/abnormal), n(\%)

Smoking, $\mathrm{n}(\%)$

Drinking, $\mathrm{n}(\%)$

Diabetes, $\mathrm{n}(\%)$

Hypertension, $\mathrm{n}(\%)$

Pathological characteristics, $\mathrm{n}(\%)$

Gross type

(protuberant/infiltrating/ulcerative)

histological type

$22(22.7 \%)$

(classical/mucous/signet-ring cell )

Differentiation

(well/moderate/poor)

$\mathrm{Ki}-67 \%($ mean $\pm S D)$

T stage, $\mathrm{n}(\%)$

(T1/T2/T3/T4)

$\mathrm{N}$ stage, $\mathrm{n}(\%)$

(N0/N1/N2)

M stage (M0/M1), n(\%)

Vascular invasion, $\mathrm{n}(\%)$

Perineural invasion, $\mathrm{n}(\%)$

LN (mean $\pm S D)$

PLN (mean \pm SD)

LNR (mean $\pm S D)$

Liver metastasis, $\mathrm{n}(\%)$

The measurement data of age, BMI, long diameter, Ki-67\%, LN, PLN, and LNR were analyzed by the method of independent $\mathrm{t}$-test. The counting data of gender, location, CEA, CA19-9, smoking, drinking, diabetes, hypertension, gross type, histological type, differentiation, $\mathrm{T} / \mathrm{N} / \mathrm{M}$ stage, and liver metastasis were analyzed by the method of chi-square test. A two-tailed $p$ value $<0.05$ indicated a statistical difference.

Performance of the clinical and pathological model
$69(71.1 \%) / 28(28.9 \%)$

$85(87.6 \%) / 12(12.4 \%)$

$26(26.8 \%)$

$9(9.3 \%)$

$44(45.4 \%)$

$28(28.9 \%) / 7(7.2 \%) / 62(63.9 \%)$

$92(94.8 \%) / 5(5.2 \%) / 0(0 \%)$

$665(96.2 \%) / 25(3.6 \%) / 1(0.1 \%)$

0.476

$55(56.7 \%) / 25(25.8 \%) / 17(17.5 \%)$

$17(18.5 \%) / 70(76.1 \%) / 5(5.4 \%)$

$74(11.1 \%) / 563(84.7 \%) / 28(4.2 \%)$

0.093

$70.11 \pm 22.60$

$68.70 \pm 20.99$

0.539

$9(9.3 \%) / 22(22.7 \%) / 56(57.7 \%) / 10(10.3 \%)$

$67(9.7 \%) / 151(21.9 \%) / 453(65.6 \%) / 20(2.9 \%)$

0.013

$p$

0.564

0.562

0.987

0.336

0.868

0.043

0.984

0.049

0.026

0.432

0.036

0.048

$92(94.8 \%) / 5(5.2 \%)$

$654(94.6 \%) / 37(5.4 \%)$

1.000

$34(35.1 \%)$

0.951

$22(22.9 \%)$

0.934

$14.16 \pm 6.28$

161(23.3\%)

0.934

$1.77 \pm 3.28$

$1.22 \pm 2.82$

0.119

$8.67 \pm 16.44$

0.041

0.242 
The LM-Clin and LM-Patho with significant clinical and pathological characteristics were constructed, respectively. The detailed parameters of models were reported in Table 2. Finally, the LM-Clin (Hosmer-Lemeshow test: $X^{2}=1.238, d f=5, p=0.941$ ) had two significant clinical characteristics of CEA and a history of hypertension, the equation was LM-Clin $=2.106+0.474{ }^{\star} \mathrm{CEA}-0.438 *$ hypertension. And the LM-Patho (Hosmer-Lemeshow test: $X^{2}=2.731, d f=7, p=0.909$ ) had two significant pathological characteristics of gross type and LNR, the equation was LM-Patho $=2.533-0.272 *$ gross- $0.012 *$ LNR. The AUCs of relevant ROCs was $0.584(95 \% \mathrm{Cl}, 0.549-0.619, p=0.004)$ for the LM-Clin and was $0.585(95 \% \mathrm{Cl}, 0.550-0.619, p=0.007)$ for the LM-Patho. The predictive efficacy of clinical and pathological models was pretty much equivalent and disappointing.

Table 2

The simple logistic models of LM-Clin, LM-Patho, and LM-Nomo.

\begin{tabular}{|c|c|c|c|c|c|c|c|}
\hline & B & S.E. & Wald & df & Sig & $\operatorname{Exp}(B)$ & $95 \% \operatorname{Exp}(B)$ \\
\hline \multicolumn{8}{|l|}{ LM-Clin } \\
\hline CEA & 0.474 & 0.239 & 3.930 & 1 & 0.047 & 1.607 & $1.005-2.568$ \\
\hline Smoking & -0.317 & 0.297 & 1.139 & 1 & 0.286 & 0.729 & $0.407-1.303$ \\
\hline Drinking & -0.325 & 0.317 & 1.049 & 1 & 0.306 & 0.723 & $0.388-1.346$ \\
\hline Hypertension & -0.438 & 0.221 & 3.918 & 1 & 0.048 & 0.645 & $0.418-0.996$ \\
\hline Constant & 2.106 & 0.174 & 147.145 & 1 & 0.000 & 8.214 & \\
\hline \multicolumn{8}{|l|}{ LM-Patho } \\
\hline Gross & -0.272 & 0.130 & 4.382 & 1 & 0.036 & 0.762 & $0.591-0.983$ \\
\hline $\mathrm{T}$ & 0.063 & 0.179 & 0.125 & 1 & 0.724 & 1.065 & $0.750-1.513$ \\
\hline LNR & -0.012 & 0.005 & 4.996 & 1 & 0.025 & 0.988 & $0.977-0.999$ \\
\hline Constant & 2.533 & 0.464 & 29.755 & 1 & 0.000 & 12.592 & \\
\hline \multicolumn{8}{|l|}{ LM-Nomo } \\
\hline Radiomic score & 1.264 & 0.180 & 49.270 & 1 & 0.000 & 3.541 & $2.488-5.040$ \\
\hline LNR & -0.013 & 0.006 & 4.844 & 1 & 0.028 & 0.987 & $0.976-0.999$ \\
\hline CEA & 0.638 & 0.256 & 6.224 & 1 & 0.013 & 1.892 & $1.147-3.123$ \\
\hline Hypertension & -0.535 & 0.236 & 5.136 & 1 & 0.023 & 0.585 & $0.368-0.930$ \\
\hline Gross & -0.251 & 0.128 & 3.781 & 1 & 0.052 & 0.779 & $0.606-1.002$ \\
\hline Constant & 0.198 & 0.461 & 0.184 & 1 & 0.668 & 1.219 & \\
\hline
\end{tabular}

\section{Performance of the radiomics and combined nomogram}

After the 12 radiomic features from three-phase CT images were extracted (Figure 2), the LM-Radio was developed. The AUC was 0.737 $(95 \% \mathrm{Cl}, 0.675-0.799)$ in the training set and was $0.709(95 \% \mathrm{Cl}, 0.617-0.801)$ in the validation set. Then the corresponding coefficients (Figure 3) were evaluated and the radiomic score was calculated.

The significant clinical and pathological characteristics integrated with radiomic score constituted the clinical-pathological-radiomic nomogram (Figure 4). The equation was LM-Nomo $=0.198+1.264 *$ radiomic score- $0.013 *$ LNR $+0.638 * C E A-0.535 *$ hypertension. The AUC of LM-Nomo was $0.757(95 \% \mathrm{Cl}, 0.726-0.787, \mathrm{p}<0.001)$. The non-significant Hosmer-Lemeshow test (Hosmer-Lemeshow test: $X^{2}=6.664, \mathrm{df}=8$, $p=0.573$ ) showed the goodness-of-fit of the model.

\section{Discussion}

Unlike MSS CRCs, the MSI-H CRCs have been proved to be associated with abundant lymphocyte infiltration, poor differentiation pattern, longer postoperative survival, predominantly occurred in the proximal colon ${ }^{[17]}$, and mucous or signet-ring cell component ${ }^{[18]}$. They may 
have a mildly better prognosis and could not benefit from 5-FU-based chemotherapy compared with patients with MSS ${ }^{[19]}$. Very few published studies have evaluated the clinical, pathological, and radiomic difference between MSI-H and MSS status in RCs. Hence, the preoperative prediction of MSI status from these fields in RCs is promising to facilitate adjuvant therapy strategies, follow-up monitoring, and management. In this analysis, we merely focused on RCs to reduce the bias between ascending, descending, and sigmoid colon. Heterogeneity of clinical and pathological manifestation of MSI-H in RCs is the rule rather than the contingency. The clinical characteristics of CEA, smoking, drinking, and hypertension history, as well as the pathological variables of gross type, T stage, and LNR had statistical

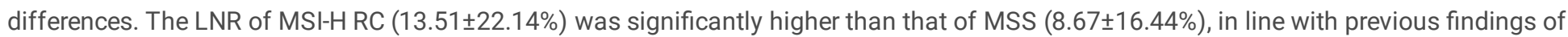
affluent lymphocyte infiltration in CRCs. And the RCs of MSI-H were founded to be easier to have a history of smoking, drinking, and hypertension. It emphasized the importance of medical history. However, they differ dramatically with CRCs, the characteristics of the location, histological type, differentiation pattern were not statistically different.

This article aimed to investigate the efficiency of the logistic models of clinic, pathology, radiomics, and the integrative nomogram to evaluate the MSI-H status. To elucidate which factors contribute to a more favorable prediction of MSI-H tumors, clinical and pathological characteristics as well as the radiomic features were analyzed in 788 patients with $97 \mathrm{MSI}-\mathrm{H}$ and $691 \mathrm{MSS}$. Since radiological images were closely connected with its pathological characteristics ${ }^{[20]}$, the quantitative radiomic features showed the potential to predict the MSI-H status of RCs in our study. Interestingly, the predictive efficacy of simple LM-Clin and LM-Patho was almost equivalent and disappointing with AUCs of 0.584 and 0.585 compared with simple LM-Radio with AUCs of 0.737 in the training set and 0.709 in the validation set, probably because the simple LM-Clin and LM-Patho could hardly discriminate MSI-H from MSS.

Therefore, an integrative nomogram including clinical characteristics, pathological characteristics, and radiomics became the most important modality to predict the MSI-H status of RCs, noninvasively. Previous studies almost exclusively focused on the evaluation of the MSI-H phenotype of CRCs, ignoring a specialized analysis of RCs. Data from the study of YT Cao et al ${ }^{[20]}$ suggested that the radiomics signature of triphasic enhanced CT was a reliable method to predict MSI in CRCs, and the clinical-radimoics nomogram including age, location, CEA, and radiomics has shown promising prediction. Our integrative clinical-pathological-radiomic nomogram including CEA, hypertension, LNR, and radiomic score was the most meaningful model in predicting MSI-H phenotype of RCs with- the highest AUC of 0.757 (95\% Cl, 0.726-0.787) than that of simple LM-Clin, LM-Patho, and LM-Radio. The $p$ values of Hosmer-Lemeshow tests of all models were non-significant, indicated the goodness-of-fit of models.

Despite some inspiring strengths, there were several limitations. First, this retrospective analysis existed several biases including singlecenter design, unbalanced sample size, and limited universality. Thus, a future multi-center supplement is necessary to validate and improve the performance of the predictive nomogram. Second, we only evaluated the tumoral radiomics to predict the MSI-H phenotype of RCs, the peri-tumoral radiomics was neglected. While peri-tumoral radiomics should be emphasized by providing additional information to better predict the MSI-H status. Third, due to the irregular shape of RCs, the bias between manual segmentation may affect the radiomic analysis, though the ICCs were calculated to reduce the intra-observer difference. An automatic approach to segment the RCs for radiomic analysis needed to be further explored.

\section{Conclusion}

In conclusion, an integrative clinical-pathological-radiomic nomogram including a history of hypertension, CEA, LNR, and radiomic score demonstrated an encouraging performance in predicting MSI-H status of RCs.

\section{Abbreviations}

Microsatellite instability: MSI

Rectal carcinoma: RC

High MSI status: MSI-H

Microsatellite stable status: MSS

The logistic model of clinical characteristics: LM-Clin

The logistic model of pathological characteristics: LM-Patho

The logistic model of radiomic features: LM-Radio 
The logistic model of a integrative nomogram: LM-Nomo

The area under curve: AUC

Lymph node metastasis ratio: LNR

Colorectal carcinoma: CRC

Mismatch repair: MMR

Low MSI status: MSI-L

Computed tomography: CT

Magnetic resonance: MR

Body mass index: BMI

Carcinoembryonic antigen: CEA

Carbohydrate antigen 19-9: CA19-9

Lymph node count: LN

Positive lymph node count: PLN

Defective mismatch repair: dMMR

Proficient mismatch repair: pMMR

Volume of interest: VOI

Intraclass correlation coefficients: ICCs

synthetic minority over-sampling technique: SMOTE

The method of variance: ANOVA

Mann-Whitney: MW

Least absolute shrinkage and selection operator: LASSO

Receiver operator curve: ROC

95\% confidence interval: $95 \% \mathrm{Cl}$

\section{Declarations}

\section{Ethics approval and consent to participate:}

This retrospective study was conducted with the permission of the Medical Ethics Committee (No. 2020QT251) and in conformity to the Declaration of Helsinki. The informed consent was waived for this retrospective study.

\section{Consent for publication:}

All the authors agreed the publication in BMC Cancer.

\section{Availability of data and materials:}

The datasets used and analyzed in this article was available from the corresponding author in reasonable request. 


\section{Competing interests and funding:}

No competing interests and funding.

\section{Authors' contribution:}

Yanqing Ma: Conceptualization, Methodology, Writing-Original Draft. Peng Yu and Jiankai Li: Writing-Review \& Editing, Supervision. Niping Song and Zi'ang Wan: : Software, Formal analysis, Data Curation. Bingchen Chen and Shiliang Tu: Validation, Resources. Yuguo Wei: Validation, Resources, statistics, and software. Hang Yuan: Conceptualization, Methodology, and editing.

\section{References}

1. Freddie, Bray, Jacques, et al. Global cancer statistics 2018: GLOBOCAN estimates of incidence and mortality worldwide for 36 cancers in 185 countries[J]. CA: A Cancer Journal for Clinicians, 2018, 68(6):384-424.

2. Siegel R L, Miller K D, Goding Sauer A, et al. Colorectal cancer statistics, 2020[J]. CA: A Cancer Journal for Clinicians, 2020, 70(3):145164.

3. Bagante F, Ruzzenente A, Sasaki K, et al. Morphologic and biologic characteristics of disease are associated with prognosis of patients with colo-rectal liver metastasis undergoing neoadjuvant chemotherapy[J]. Hpb, 2018, 20(189-190.

4. Trojan J, Stintzing S, Haase O, et al. Complete pathological response after neoadjuvant short-course immunotherapy with ipilimumab and nivolumab in locally advanced MSI-H/dMMR rectal cancer[J]. The Oncologist, 2021.

5. Boland C R,Goel A. Microsatellite instability in colorectal cancer[J]. Gastroenterology, 2010, 138(6):2073-2087.

6. Fan S, Li X, Cui X, et al. Computed Tomography-Based Radiomic Features Could Potentially Predict Microsatellite Instability Status in Stage II Colorectal Cancer: A Preliminary Study[J]. Academic Radiology, 2019, 26(12):1633-1640.

7. Kloor M, Staffa L, Ahadova A, et al. Clinical significance of microsatellite instability in colorectal cancer[J]. Langenbeck's Archives of Surgery, 2014, 399(1):23-31.

8. Mayerhoefer M E, Materka A, Langs G, et al. Introduction to Radiomics[J]. J Nucl Med, 2020, 61(4):488-495.

9. Pei Q, Yi X, Chen C, et al. Pre-treatment CT-based radiomics nomogram for predicting microsatellite instability status in colorectal cancer[J]. European Radiology, 2021, 1-11.

10. Zhang W, Yin H, Huang Z, et al. Development and validation of MRI-based deep learning models for prediction of microsatellite instability in rectal cancer[J]. Cancer Med, 2021, 10(12):4164-4173.

11. Li Z, Dai H, Liu Y, et al. Radiomics Analysis of Multi-Sequence MR Images For Predicting Microsatellite Instability Status Preoperatively in Rectal Cancer[J]. Frontiers in Oncology, 2021, 7(11):697497.

12. Zhang W, Huang Z, Zhao J, et al. Development and validation of magnetic resonance imaging- based radiomics models for preoperative prediction of microsatellite instability in rectal cancer[J]. Annals of Translational Medicine, 2021, 9(2):134-146.

13. Huang Z, Zhang W, He D, et al. Development and validation of a radiomics model based on T2WI images for preoperative prediction of microsatellite instability status in rectal cancer: Study Protocol Clinical Trial (SPIRIT Compliant)[J]. Medicine, 2020, 99(10):e19428.

14. Umar A, Boland C R, Terdiman J P, et al. Revised Bethesda Guidelines for hereditary nonpolyposis colorectal cancer (Lynch syndrome) and microsatellite instability[J]. Journal of the National Cancer Institute, 2004, 96(4):261-268.

15. Büsing K A, Kilian Ak Fau - Schaible T, Schaible T Fau - Debus A, et al. Reliability and validity of MR image lung volume measurement in fetuses with congenital diaphragmatic hernia and in vitro lung models[J]. RADIOLOGY, 2008, 246(2):553-561.

16. Koivu A, Sairanen M, Airola A, et al. Synthetic minority oversampling of vital statistics data with generative adversarial networks[J]. J Am Med Inform Assoc, 2020, 27(11):1667-1674.

17. Meng W J, Sun X F, Tian C, et al. Microsatellite instability did not predict individual survival in sporadic stage II and III rectal cancer patients[J]. Oncology, 2007, 72(1-2):82-88.

18. Kawakami H, Zaanan A,Sinicrope F A. Microsatellite instability testing and its role in the management of colorectal cancer[J]. Curr Treat Options Oncol, 2015, 16(7):30.

19. de la Chapelle A,Hampel H. Clinical relevance of microsatellite instability in colorectal cancer[J]. Journal of Clinical Oncology, 2010, 28(20):3380-7.

20. Cao Y, Zhang G, Zhang J, et al. Predicting Microsatellite Instability Status in Colorectal Cancer Based on Triphasic Enhanced Computed Tomography Radiomics Signatures: A Multicenter Study[J]. Front Oncol, 2021, 10(11):687771. 
Figures
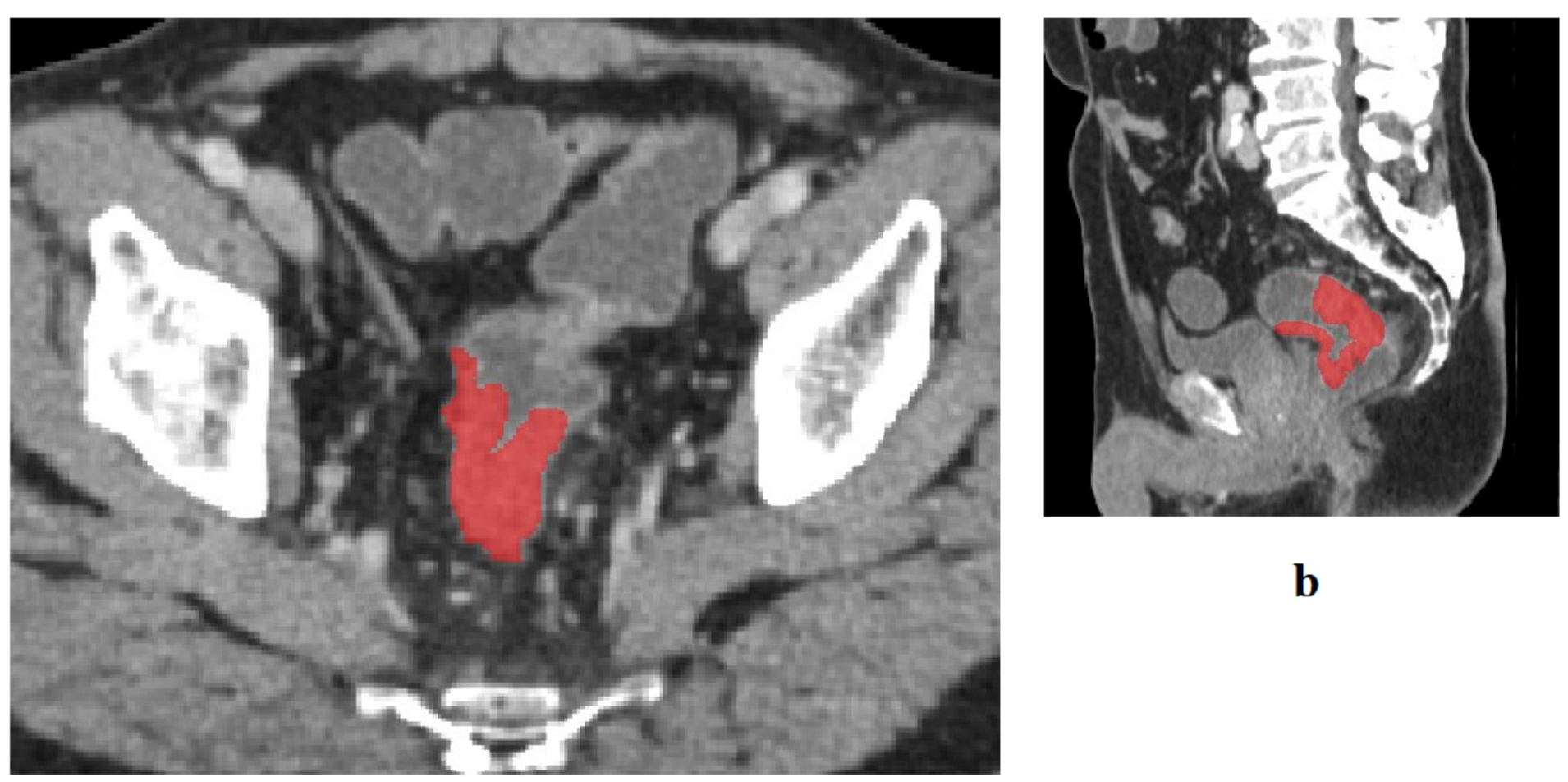

\section{b}

$\mathbf{a}$

\section{Figure 1}

The VOIs were manually segmented in the software of "itk-SNAP". Figure 1a showed the VOIs segmentation in the axial image. Figure 1b showed the VOls segmentation in the sagittal image. 

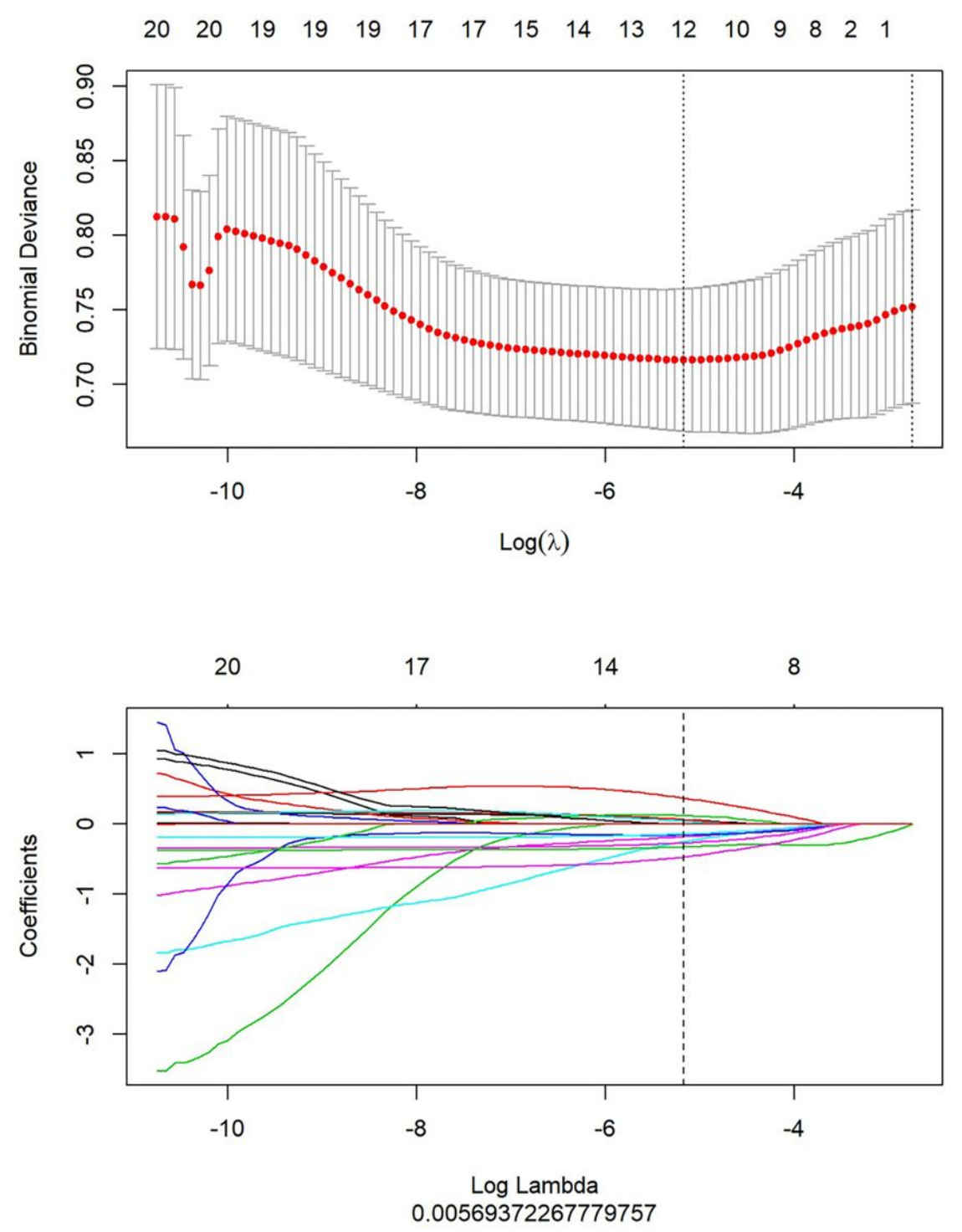

Figure 2

To the radiomic analysis, after the method of LASSO, there were 12 radiomic features extracted. 


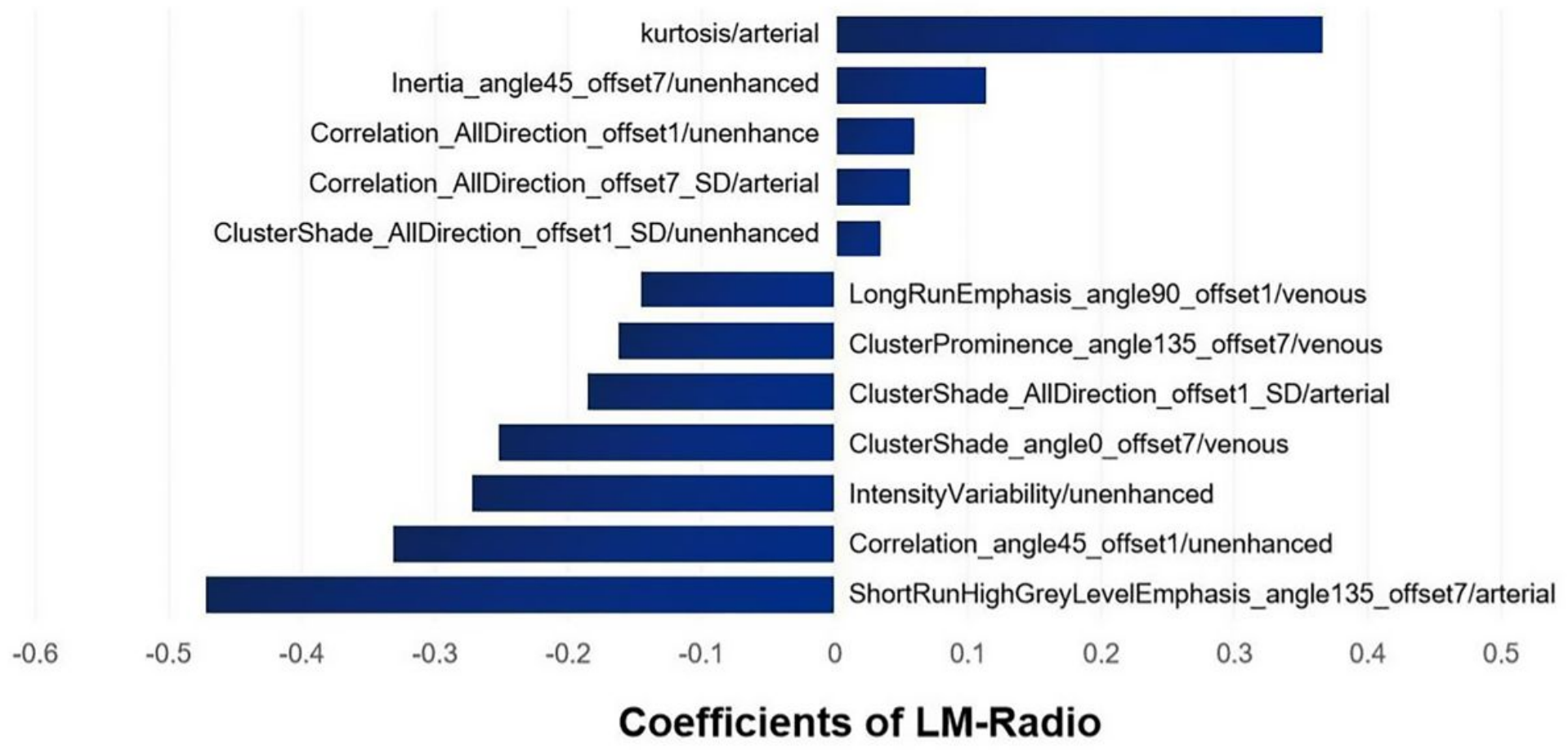

\section{Figure 3}

The coefficients of 12 optimal radiomic features in LM-Radio were listed.

Points
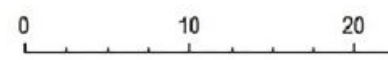
30 40
1 50 60 70 80 90 100

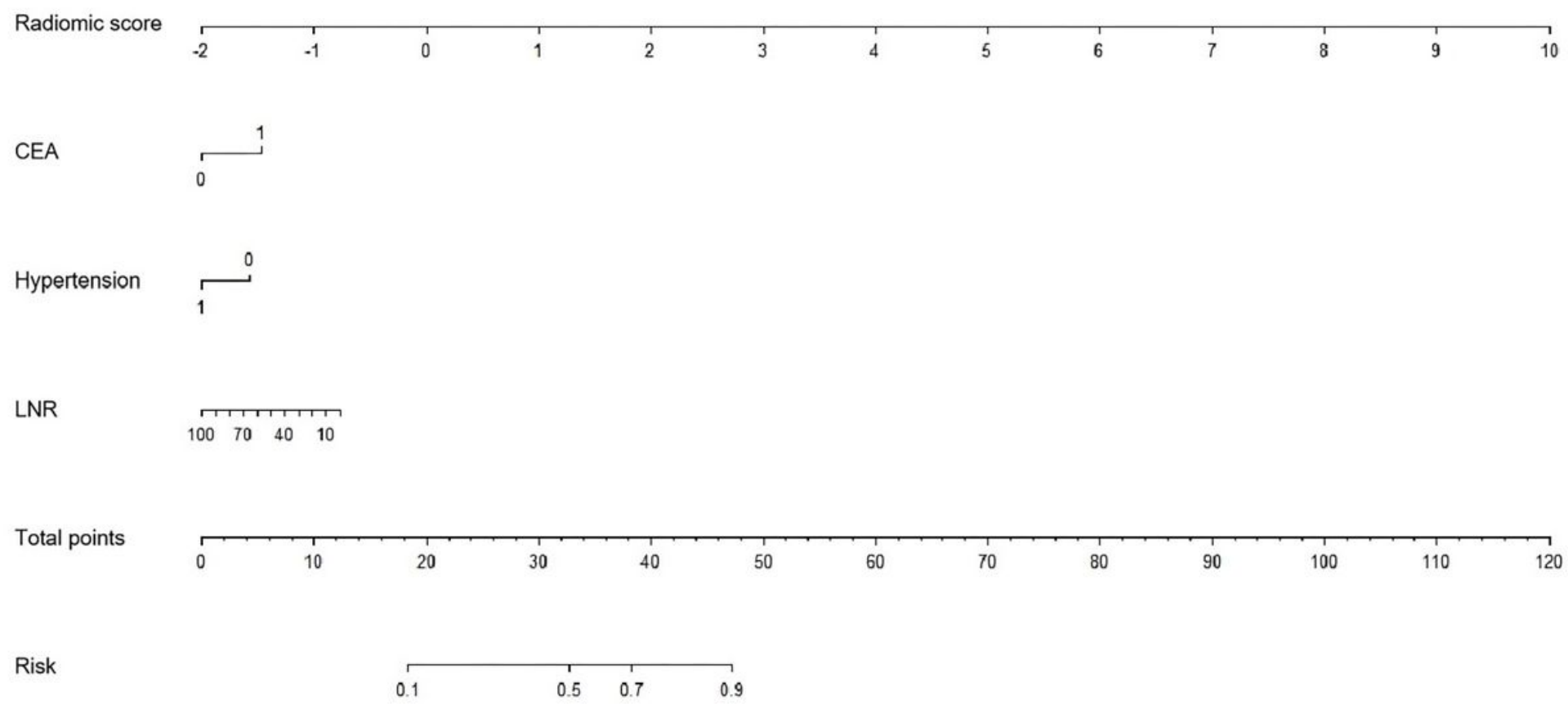

Figure 4

The integrative clinical-pathological-radiomic nomogram including variables of CEA, hypertension, LNR, and radiomic score was developed.

\section{Supplementary Files}


This is a list of supplementary files associated with this preprint. Click to download.

- SupplementMaterial.doc 